\title{
A BOUND FOR THE HILBERT-SCHMIDT NORM OF GENERALIZED COMMUTATORS OF NONSELF-ADJOINT OPERATORS
}

\author{
MiCHAEL GiL'
}

Abstract. Let $A, \tilde{A}$ and $B$ be bounded linear operators in a Hilbert space, and $f(z)$ be a function regular on the convex hull of the union of the spectra of $A$ and $\tilde{A}$. Let $S N_{2}$ be the ideal of Hilbert-Schmidt operators. In the paper, a sharp estimate for the Hilbert-Schmidt norm of the commutator $f(A) B-B f(\tilde{A})$ is established, provided $A B-B \tilde{A} \in S N_{2}, A-A^{*} \in S N_{2}$ and $\tilde{A}-$ $\tilde{A}^{*} \in S N_{2}$. Here the star means the adjointness. Our results are new even in the finite dimensional case.

Mathematics subject classification (2010): 47B47, 47A30, 47B10, 47A56, 47A55.

Keywords and phrases: Generalized commutator, inequality, nonself-adjoint operators, operator function, Hilbert-Schmidt norm.

\section{REFERENCES}

[1] R. Bhatia And F. Kittaneh, Some inequalities for norms of commutators, SIAM J. Matrix Anal. Appl. 18 (1997), 258-263.

[2] M. Birman, AND M. SOlOMYAK, Double operator integrals in a Hilbert space, Integral Equations Operator Theory 47, no. 2, (2003), 131-168.

[3] K. N. Boyadzhiev, Some inequalities for generalized commutators, Publ. RIMS, Kyoto University, 26, no. 3 (1990), 521-527.

[4] K. N. BoyAdZHIEV, Norm estimates for commutators of operators, J. London Math. Soc. 57 (2) (1998), 739-745.

[5] M. I. GIL', Operator Functions and Localization of Spectra, Lecture Notes In Mathematics, vol. 1830, Springer-Verlag, Berlin, 2003.

[6] M. I. GIL', Perturbations of functions of operators in a Banach space, Math. Phys. Anal. Geom. 13, no. $1,(2010)$ 69-82.

[7] M. I. GIL', Perturbations of operator functions in a Hilbert Space, Communications in Mathematical Analysis 13, no. 2, (2012) 108-115.

[8] I. C. GohberG, AND M. G. KREIN, Introduction to the Theory of Linear Nonselfadjoint Operators, Trans. Mathem. Monographs, v. 18, Amer. Math. Soc., Providence, R. I, 1969.

[9] P. Halmos, Hilbert Space Problem Book, Second edition, Springer Verlag, New York, 1982.

[10] F. KitTAneH, Norm Inequalities for commutators of $G_{1}$ operators, Complex Anal. Oper. Theory 10, (2016) 109-114.

[11] V. PELLER, Hankel operators in perturbation theory of unbounded self-adjoint operators, in the book Analysis and Partial Differential Equations, Lecture Notes in Pure and Appl. Math. 122, Marcel Dekker, New York, 1990, pp. 529-544. 